\title{
Effect of collisions on drift instabilities in a field reversed configuration under conditions of magneto-inertial fusion
}

\author{
Guanqiong Wang, ${ }^{1}$ Xiaoguang Wang, ${ }^{1}$ Xianjun Yang, ${ }^{1,2}$ and Jan Weiland ${ }^{3}$ \\ ${ }^{1}$ Center for Fusion Energy Science and Technology, China Academy of Engineering Physics, \\ Beijing 100088, China \\ ${ }^{2}$ Institute of Applied Physics and Computational Mathematics, Beijing 100094, China \\ ${ }^{3}$ Chalmers University of Technology and EURATOM-VR Association, Gothenburg, Sweden
}

(Received 19 October 2016; accepted 15 May 2017; published online 9 June 2017)

Drift instabilities in a field reversed configuration are studied under conditions of magneto-inertial fusion (MIF). Specifically, the collisional effect is taken into account because of high-density plasmas in MIF where the drift wave frequency is smaller than the electron-ion collision frequency. Dispersion relations are based on the two fluid equations including the collisional terms; meanwhile, the electromagnetic effect is also considered due to high $\beta$ values ( $\beta$ is the ratio of plasma pressure to magnetic pressure). It is found that in the limit of low $\beta$, the behavior of instabilities described by the dispersion relations in the present paper would become like drift instabilities in tokamaks, where $\beta \sim 0.1$. Therefore, in the MIF case, electromagnetic drift instabilities could be driven by electron-ion collisions due to the charge separation effect. The collisions also bring the phase difference between the perturbed density and the potential perturbation, which is significant for the particle transport. Published by AIP Publishing. [http://dx.doi.org/10.1063/1.4985079]

\section{INTRODUCTION}

In contrast to the traditional approaches to fusion energy including the magnetic fusion energy (MFE) ${ }^{1}$ and the inertial confinement fusion (ICF), ${ }^{2}$ the magneto-inertial fusion $(\mathrm{MIF})^{3,4}$ is viewed as a new "intermediate" way to fusion energy because the density regime and time scale of MIF are intermediate between MFE and ICF. In the MIF scheme, an appropriate magnetic field should be embedded in a plasma target, and the field reversed configuration (FRC) ${ }^{5}$ is one candidate magnetic configuration. The FRC is an elongated compact torus (CT) with predominantly poloidal fields and zero toroidal fields (Fig. 1). The magnetic surface of FRC is robust and closed, so that the topology of the magnetic field can be retained during implosion and the separatrix can insulate the fuel from the imploding wall or "pusher". Due to the limitation of the present driver capability, the FRC plasma target must satisfy some conditions in different phases. ${ }^{6}$ Table I shows typical values of various parameters for a dense FRC. In order to improve the confinement of the FRC target and satisfy the requirement for MIF, it is necessary to understand the transport mechanism.

To some extent, the FRC transport is unique due to the very high $\beta$ and the magnetic field nulls. The value of $\beta_{0}$ averaged over the plasma volume is about $0.5-0.9,{ }^{7}$ where $\beta_{0}=2 \mu_{0} p / B_{e}^{2}$ is the ratio of plasma pressure to the external magnetic field pressure. The understanding of FRC transport has advanced considerably in the past 30 years; particle, ${ }^{8,9}$ magnetic flux, and energy confinement ${ }^{10}$ are well identified as anomalous; in other words, certain instabilities induce the turbulence which causes the anomalous transport in FRCs. In the beginning, the lower hybrid drift (LHD) instability, ${ }^{11}$ which is electrostatic $(\delta B=0)$ and flute like $\left(k_{\|}=0\right)$ with wave numbers $k \sim 1 / \rho_{e}$, was considered as the most linearly instable and studied in a lot of works. ${ }^{12-15}$ Specifically, the electromagnetic LHDI was investigated by using a simple two-fluid theory in Refs. 16 and 17, and this analysis was extended by adding collisions and the non-local effect ${ }^{18}$ in order to explain the anomalous resistivity in the magnetic reconnection experiment ${ }^{19}$ (MRX) where a Harris equilibrium is applied, which is significantly different from the FRC equilibrium. ${ }^{5,10}$ However, fluctuation measurements in FRC experiments ${ }^{20,21}$ did not verify LHD transport theories. Then, it was proved that LHD waves decay strongly into lower wave-number modes ${ }^{22}$ which usually stay in the lowfrequency drift wave regime. This indicates that other instabilities should be considered. Then, electron temperature gradient (ETG) driven electromagnetic modes were studied in Ref. 23. ETG instability parameters at $L_{n} \sim \rho_{i}$ are as follows: $k>1 / \rho_{e}$ and $\gamma \sim \Omega_{c i}\left(L_{n}\right.$ is the density gradient scale length, $\rho_{e}$ is the electron gyroradii, and $\gamma$ is the growth rate). Note that small parallel wave number $k_{\|} \neq 0$ is needed for this instability which propagates in the direction opposite to the LHD.

So far, drift instabilities have been proposed to be responsible for the anomalous transport in FRCs. Note that drift instabilities mentioned in this paper are in the lowfrequency range $\left(\omega \ll \Omega_{c i}\right)$, rather than in the lower hybrid frequency range. Both the kinetic theory ${ }^{7,14}$ and fluid treatment ${ }^{24,25}$ are used to address the electromagnetic drift instabilities. In addition, the gyrokinetic particle simulation of turbulent transport in FRC is presented in Ref. 8. Lowfrequency drift dissipative modes are studied in Ref. 14 where both the temperature and the magnetic field are uniform, and the Krook model is used to introduce the effect of collisions; however, the condition $\nu_{e i} \ll \Omega_{c i}$ is needed which is not justified in the parameter regime of MIF. In Ref. 7, the dispersion equation is based on the set of Vlasov-Maxwell equations taking into account the nonadiabatic response of 


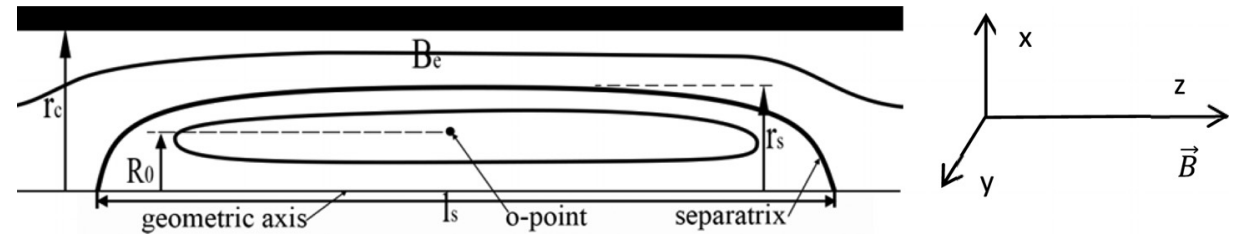

FIG. 1. Anatomy of a FRC and the local coordinate frame of calculations for drift waves.

TABLE I. Parameters of a typical MIF target (FRC) in different states. State 1 and State 2 correspond to the initial and intermediate stages of the implosion, respectively. State 3 presents a late stage (near the stagnation point). Here, the parameters include density $n$, temperature $T$, magnetic field $B$, separatrix radius $r_{s}$, length of separatrix $l_{s}$, electron-ion collision frequency $\nu_{e i}$, cyclotron frequency of electrons and ions $\Omega_{c e}$ and $\Omega_{c i}$, thermal gyroradii of ions $\rho_{i}$, and the ion mean free path $\lambda_{i}$.

\begin{tabular}{|c|c|c|c|c|c|c|c|c|c|c|}
\hline & $\mathrm{n}\left(\mathrm{m}^{-3}\right)$ & $\mathrm{T}(\mathrm{eV})$ & $\mathrm{B}(\mathrm{T})$ & $r_{s}(\mathrm{~cm})$ & $l_{s}(\mathrm{~cm})$ & $\nu_{e i}\left(\mathrm{~s}^{-1}\right)$ & $\Omega_{c e}\left(\mathrm{~s}^{-1}\right)$ & $\Omega_{c i}\left(\mathrm{~s}^{-1}\right)$ & $\rho_{i}(\mathrm{~cm})$ & $\lambda_{i}(\mathrm{~cm})$ \\
\hline State 1 & $10^{23}$ & $0.3 \times 10^{3}$ & 2 & 2.5 & 15 & $6.96 \times 10^{8}$ & $3.52 \times 10^{11}$ & $9.65 \times 10^{7}$ & 0.12 & 1.04 \\
\hline State 2 & $10^{25}$ & $1 \times 10^{3}$ & 20 & 0.75 & 4.5 & $1.10 \times 10^{10}$ & $3.52 \times 10^{12}$ & $9.65 \times 10^{8}$ & 0.023 & 0.121 \\
\hline State 3 & $10^{27}$ & $5 \times 10^{3}$ & 200 & 0.25 & 1.5 & $9.92 \times 10^{10}$ & $3.52 \times 10^{13}$ & $9.65 \times 10^{9}$ & $5.1 \times 10^{-3}$ & 0.0299 \\
\hline
\end{tabular}

both ions and electrons; however, the collisional effect is not included. In Ref. 25, a two-fluid theory is used to obtain the dispersion relation and estimate the transport coefficient in the high-density case relevant to MIF. Although the high collisionality is considered in Ref. 25, there are no collisional terms in fluid equations, or the collisional effect is not studied in detail.

Actually, in MIF relevant parameters, the electron-ion collision frequency is larger than ion cyclotron frequency, i.e., $\nu_{e i}>\Omega_{c i}$, because of the high density. So that the effect of collisions should be investigated carefully. Meanwhile, the electromagnetic effect may be important due to high $\beta$. In this work, the effect of collisions on electromagnetic drift instabilities will be studied.

This paper is organized as follows. Section II derives the dispersion relation of drift waves by using two fluid equations including collisional terms. In Sec. III, we present and discuss the results obtained from the numerical solution of the dispersion relation. At last, the conclusions (Sec. IV) are given.

\section{PHYSICAL MODEL}

In the derivations, the local Cartesian coordinate is applied: all gradients are parallel or antiparallel to the $\mathrm{x}$-axis (directed from the plasma core to edge regions), the equilibrium magnetic field lines are along the $\mathrm{z}$-axis, and the $\mathrm{y}$-axis is perpendicular to both $\mathrm{x}$ and $\mathrm{z}$. We also assumed that the modes propagate in the $\mathrm{y}-\mathrm{z}$ plane, and the space and time dependence of the perturbed variables is given by $\exp \left[i\left(k_{y} y+k_{\|} z-\omega t\right)\right]$. The objective is to obtain a dispersion relation of drift waves including the effects of collisions, perturbed magnetic field, field line curvature, and transversal nonuniformity of the magnetic field.

\section{A. Ion equations}

\section{Ion continuity equation}

$$
\frac{\partial n_{i}}{\partial t}+\nabla \cdot\left(n_{i} \mathbf{v}_{* \mathbf{i}}+n_{i} \mathbf{v}_{E}+n_{i} \mathbf{v}_{p i}+n_{i} \mathbf{v}_{\pi i}\right)=0 .
$$

E cross B drift velocity is given by

$$
\mathbf{v}_{\mathbf{E}}=\mathbf{E} \times \hat{b} / B=\hat{b} \times(\nabla \phi+\partial \mathbf{A} / \partial t) / B
$$

when the electromagnetic effect is included, the perturbed field can be defined as follows: electric field is $E$ $=-\nabla \phi-\partial \mathbf{A} / \partial t$ and magnetic field is $\mathbf{B}=\nabla \times \mathbf{A}$, where $\phi$ represents the perturbed scalar electric potential and $\mathbf{A}$ is the perturbed vector potential. The Coulomb gauge $\nabla \cdot \mathbf{A}$ $=0$ is used.

The ion diamagnetic drift is

$$
\mathbf{v}_{* i}=\frac{\hat{b} \times \nabla p_{i}}{Z_{i} e n_{i} B}=\frac{\hat{b} \times \nabla p_{i}}{Z_{i} e n_{i} B_{0}}\left(1-\frac{\delta B_{/ /}}{B_{0}}\right),
$$

where $\delta B_{\|}$is the parallel component of the perturbed magnetic field, $B_{0}$ represents the background magnetic field, $\hat{b}$ $=\mathbf{B} / B$ is the unit vector along the magnetic field line.

The polarization drift velocity is

$$
v_{p i}=\frac{d \mathbf{E}}{d t} /\left(B \Omega_{i}\right),
$$

where $\Omega_{i}=Z_{i} e B / m_{i}$ is the ion cyclotron frequency.

The ion drift due to the off-diagonal elements of the stress tensor $\ddot{\pi}_{i}$ is

$$
\mathbf{v}_{\pi i}=\frac{\hat{b} \times \nabla \vec{\pi}}{Z_{i} e n_{i} B} .
$$

The equations for the divergence of the drifts are ${ }^{26}$

$$
\begin{gathered}
\nabla \cdot \delta\left(n_{i} \mathbf{v}_{* i}\right)=\frac{1}{T_{i}} \mathbf{v}_{D i} \cdot \nabla \delta p_{i}+\frac{\mu_{0} n_{i}}{B_{0}^{2}} \mathbf{v}_{* i} \cdot \nabla \delta\left(p_{i}+p_{e}\right), \\
\nabla \cdot \delta\left(n_{i} \mathbf{v}_{\mathbf{p i}}+n_{i} \mathbf{v}_{\pi i}\right) \approx-i n_{i} k_{y}^{2} \rho_{s}^{2} Z_{i}\left(\omega-\omega_{* i T}\right) \frac{e \phi}{T_{e}},
\end{gathered}
$$

where ion gyro-radius $\rho_{s}=c_{s} / \Omega_{i}$, ion sound speed $c_{s}=\sqrt{T_{e} / m_{i}}, \omega_{* i T}=\omega_{* i}\left(1+\eta_{i}\right)$, the ion diamagnetic drift frequency $\omega_{* i}=-k_{y} T_{i}\left|\nabla n_{i}\right| /\left(Z_{i} e n_{i} B\right), Z_{i}$ is the ion charge number, and $\eta_{i}$ is the ratio of the density gradient scale length to the temperature gradient scale length $\eta_{i} \equiv L_{n} / L_{T i}$ 
$=n_{i}\left|\nabla T_{i}\right| /\left(T_{i}\left|\nabla n_{i}\right|\right)$. Compared with the electrostatic case in Ref. 26, the second term on the right-hand side of Eq. (6) is introduced due to the electromagnetic effect. Equation (7) indicates the finite Larmor radius (FLR) effect which is included through combining the polarization drift with the stress tensor drift, resulting in the cancelation of the convective diamagnetic part of the polarization drift. ${ }^{26}$

After linearizing Eq. (1), we get the density perturbation

$$
\begin{aligned}
& \left(\omega_{D i}-\omega+\frac{\beta_{t}}{2} \omega_{* i T}\right) \frac{\delta n_{i}}{n_{i}}+\left[\omega_{* e}-\omega_{D e}-k_{y}^{2} \rho_{s}^{2} Z_{i}\left(\omega-\omega_{* i T}\right)\right] \\
& \quad \times \frac{e \phi}{T_{e}}-\left[\omega_{* e}-\omega_{D e}-k_{y}^{2} \rho_{s}^{2} Z_{i}\left(\omega-\omega_{* i T}\right)\right] \omega \frac{e A_{y}}{T_{e} k_{y}} \\
& +\left(\omega_{D i}+\frac{\beta_{i}}{2} \omega_{* i T}\right) \frac{\delta T_{i}}{T_{i}}+\frac{\beta_{e}}{2} \omega_{* i T} \frac{\delta T_{e}}{T_{e}}=0
\end{aligned}
$$

where the local beta parameter $\beta_{t}=2 \mu_{0}\left(p_{i}+p_{e}\right) / B_{0}^{2}$ is the sum of ion pressure ratio $\beta_{i}$ and electron pressure ratio $\beta_{e}$. The ion magnetic drift frequency can be presented as

$$
\omega_{D i}=\omega_{* i}\left(\frac{L_{n}}{L_{s}}-\frac{L_{n}}{L_{B}}\right),
$$

where $L_{B}=B /|\nabla B|$ is the scale of the magnetic field gradient and $1 / L_{S}$ is the curvature of the magnetic field. Note that the gradient of magnetic field is opposite to the density gradient in FRCs.

\section{Ion energy balance equation}

$$
\begin{aligned}
& \frac{3}{2} n_{i}\left(\frac{\partial}{\partial t}+\mathbf{v}_{\mathbf{i}} \cdot \nabla\right) T_{i}+n_{i} T_{i} \nabla \cdot \mathbf{v}_{\mathbf{i}}=-\nabla \cdot \mathbf{q}_{* i} \\
& \quad=\frac{5}{2} n_{i}\left(\mathbf{v}_{* i}-\mathbf{v}_{\mathbf{D i}}\right) \cdot \nabla T_{i},
\end{aligned}
$$

where the diamagnetic heat flow $\mathbf{q}_{* i}=\frac{5}{2} \frac{n_{i} T_{i}}{m_{i} \Omega_{j}}\left(\hat{\mathbf{b}} \times \nabla T_{i}\right)$, note that we just keep the reactive term comparing the heat flow in Braginskii equations. When the continuity equation is used, the first convective diamagnetic part of $\nabla \cdot \mathbf{q}_{* i}$ cancels with other convective diamagnetic terms, and the linearized temperature perturbation is now

$$
\begin{gathered}
\frac{2}{3} \omega \frac{\delta n_{i}}{n_{i}}+\omega_{* e}\left(\eta_{i}-\frac{2}{3}\right) \frac{e \phi}{T_{e}}-\omega_{* e}\left(\eta_{i}-\frac{2}{3}\right) \omega \frac{e A_{y}}{k_{y} T_{e}} \\
-\left(\omega-\frac{5}{3} \omega_{D i}\right) \frac{\delta T_{i}}{T_{i}}=0 .
\end{gathered}
$$

\section{B. Electron equations}

Parallel electron motion gives

$$
\begin{aligned}
m_{e}\left(\frac{\partial}{\partial t}+\mathbf{v} \cdot \nabla\right) v_{/ / e}= & e \hat{b} \cdot(\nabla \phi+\partial \mathbf{A} / \partial t)-e \hat{b} \cdot\left(\mathbf{v}_{* e} \times\right. \\
& -\frac{1}{n_{e}} R_{e i}-\frac{1}{n_{e}} \hat{b} \cdot \nabla p_{e}
\end{aligned}
$$

where $R_{e i}=J_{/ /} m_{e} \nu_{e i} / 1.96 e$ is the momentum gained by electrons through collisions with ions in which the parallel current $J_{/ /} \approx J_{/ / e}=-e n_{e} \delta v_{/ / e}$. Then, ignoring electron inertia, we have

$$
\begin{aligned}
& k_{/ /} \frac{e \phi}{T_{e}}-k_{/ /} \omega_{* e T} \frac{e A_{y}}{k_{y} T_{e}}+\left(\omega_{* e T}-\omega\right) \frac{e A / /}{T_{e}}+i \frac{m_{e} \nu_{e i}}{1.96 T_{e}} \delta v_{/ / e} \\
& -k_{/ /} \frac{\delta n_{e}}{n_{e}}-k_{/ /} \frac{\delta T_{e}}{T_{e}}=0
\end{aligned}
$$

where $\nu_{e i}$ is the electron-ion collision frequency.

Along the field lines, electrons are assumed to be isothermal; thus,

$$
\left(\mathbf{B}_{0}+\delta \mathbf{B}\right) \cdot \nabla\left(T_{e 0}+\delta T_{e}\right)=0
$$

and after linearizing Eq. (14), one has

$$
\eta_{e} \omega_{* e} \frac{e A_{/ /}}{k_{/ /} T_{e}}-\eta_{e} \omega_{* e} \frac{e A_{y}}{k_{y} T_{e}}-\frac{\delta T_{e}}{T_{e}}=0 .
$$

Then, linearizing the electron continuity equation

$$
\frac{\partial n_{e}}{\partial t}+\nabla \cdot\left[n_{e}\left(\mathbf{v}_{\mathbf{E}}+\mathbf{v}_{* e}+\mathbf{v}_{/ / e}\right)\right]=0
$$

one gets

$$
\begin{aligned}
& \left(-\omega+\omega_{D e}+\frac{\beta_{t}}{2} \omega_{* e T}\right) \frac{\delta n_{e}}{n_{e}}+\left(\omega_{* e}-\omega_{D e}\right) \frac{e \phi}{T_{e}} \\
& -\left(\omega_{* e}-\omega_{D e}\right) \omega \frac{e A_{y}}{k_{y} T_{e}}+\left(\omega_{D e}+\frac{\beta_{e}}{2} \omega_{* e T}\right) \frac{\delta T_{e}}{T_{e}} \\
& +\frac{\beta_{i}}{2} \omega_{* e T} \frac{\delta T_{i}}{T_{i}}+k_{/ /} \delta v_{/ / e}=0 .
\end{aligned}
$$

Here, it is seen that the ion temperature perturbation has an influence on the electron density response because of high beta. Although the electron-ion collision frequency is very high, it is still much less than the electron cyclotron frequency. Thus, the effect of collisions on the electron perpendicular motion could be neglected.

\section{Ampere's law}

$$
\nabla \times(\nabla \times \mathbf{A})=\mu_{o} \mathbf{J}
$$

For the parallel component $-\nabla^{2} A_{/ /}=\mu_{o} J_{/ /} \approx-\mu_{o} e n_{e} \delta v_{/ / e}$, with the assumption $k_{\perp} \gg k_{\|}$we have

$$
\delta v_{/ / e}+k_{\perp}^{2} \rho_{s}^{2} v_{A}^{2} \frac{e A / /}{T_{e}}=0
$$

where Alfven speed $v_{A}=B / \sqrt{\mu_{0} n m_{i}}$.

In the perpendicular direction, the perturbed current is given by 


$$
\begin{aligned}
\delta \mathbf{J}_{\perp} & =Z_{i} e n_{i} v_{* i}-e n_{e} v_{* e} \\
& =\frac{\hat{b} \times \nabla\left(\delta p_{i}+\delta p_{e}\right)}{B_{0}}-\frac{\hat{b} \times \nabla\left(p_{i}+p_{e}\right)}{B_{0}} \frac{\delta B_{/ /}}{B_{0}}
\end{aligned}
$$

so that the perpendicular component of Eq. (18) yields

$$
k_{y} A_{y}+C O E \cdot k_{/ /} A_{/ /}=0,
$$

where $C O E=-\left(i k_{x}-\frac{\beta_{t}}{2 L_{p t}}\right) / \frac{\beta_{t}}{2 L_{p t}}$, the total pressure gradient scale length is $L_{p t}=-p_{t} /\left(\partial p_{t} / \partial x\right)$, and $p_{t}=p_{i}+p_{e}$.
However, with the assumption that the modes propagate in the $\mathrm{y}-\mathrm{z}$ plane, COE should be equal to 1 .

\section{Dispersion relation}

From Eqs. (8), (11), (13), (15), (17), (19), and (21), one can obtain the set of linear algebraic equations of variables $\delta \mathrm{n}, \phi, A_{y}, \delta T_{i}, \delta T_{e}, A_{\|}$, and $\delta v_{\| e}$. Here, we have used the neutrality condition, i.e., $\delta \mathrm{n}=\delta n_{e}=\delta n_{i}$. After the equations are normalized by $\omega_{* e}$, the matrix of this system becomes

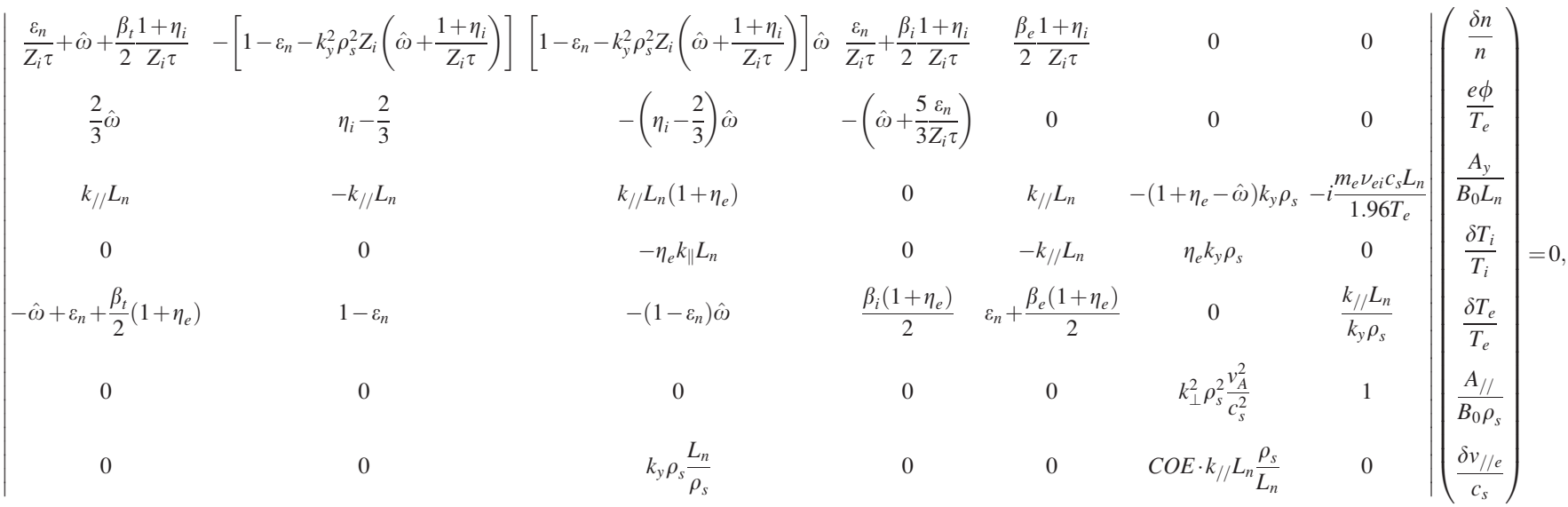

where $\varepsilon_{n}=\omega_{D e} / \omega_{* e}=L_{n} / L_{s}-L_{n} / L_{B}$ indicates the effects of curvature and gradient of the magnetic field, $\tau=T_{e} / T_{i}$ is the ratio of electron temperature to ion temperature, and $\hat{\omega}=\omega / \omega_{* e}$ represents normalized mode frequency which is complex. When the determination of system (22) equals zero, we could obtain the dispersion equation and the dependencies $\omega\left(k_{\perp}, k_{/ /}\right)$.

\section{RESULTS AND DISCUSSION}

\section{A. Analytical}

In the parameter range of drift waves, usually we assume $k_{/ /} \ll k_{\perp}$. Therefore, as mentioned above the wave vector along the $\mathrm{x}$-axis is neglected, i.e., $\mathrm{COE}=1$. In the MIF case, note that $v_{A}^{2} / c_{s}^{2} \sim 2 / \beta_{e} \sim 1, D_{e i} \sim 0.1$, and $k_{y} \rho_{s} \geq 1$. Thus, after the diagonalization of determination of system (22), the perturbed electron density response is

$$
\frac{\delta n_{e}}{n_{e}}=\frac{\left(1-\varepsilon_{n}\right)\left[i D_{e i} k_{y} \rho_{s} \frac{v_{A}^{2}}{c_{s}^{2}}-(1-\hat{\omega})\right]+\eta_{e}\left[\varepsilon_{n}+\frac{\beta_{e}\left(1+\eta_{e}\right)}{2}\right]+\left(\eta_{i}-\frac{2}{3}\right) \frac{\beta_{i}\left(1+\eta_{e}\right)}{2\left(\hat{\omega}+\frac{5}{3} \frac{\varepsilon_{n}}{Z_{i} \tau}\right)}\left[i D_{e i} k_{y} \rho_{s} \frac{v_{A}^{2}}{c_{s}^{2}}-(1-\hat{\omega})\right]}{\left[\hat{\omega}-\frac{\beta_{t}}{2}\left(1+\eta_{e}\right)-\varepsilon_{n}\right]\left[i D_{e i} k_{y} \rho_{s} \frac{v_{A}^{2}}{c_{s}^{2}}-(1-\hat{\omega})\right]+\eta_{e}\left[\varepsilon_{n}+\frac{\beta_{e}\left(1+\eta_{e}\right)}{2}\right]-\frac{2 \hat{\omega}}{3} \frac{\beta_{i}\left(1+\eta_{e}\right)}{2\left(\hat{\omega}+\frac{5}{3} \frac{\varepsilon_{n}}{Z_{i} \tau}\right)}\left[i D_{e i} k_{y} \rho_{s} \frac{v_{A}^{2}}{c_{s}^{2}}-(1-\hat{\omega})\right]} \frac{e \phi}{T_{e}},
$$

where the terms including $\beta_{i}$ represent the contribution from ions, which are introduced when the ion temperature perturbation in the electron continuity equation is substituted. The normalized collisionality $D_{e i}$ appears in the imaginary part, so that it causes the phase difference between the perturbed density and the electrostatic potential perturbation, which is critical for the transport caused by drift instabilities. It is easy to confirm that Eq. (23) gives a Boltzmann distribution in the limit of infinite collisionality.

\section{B. Numerical}

In this work, we focus on the State 1 mentioned in Table I, since it is unclear whether the drift instabilities have negative effects on a dense FRC performance before the 


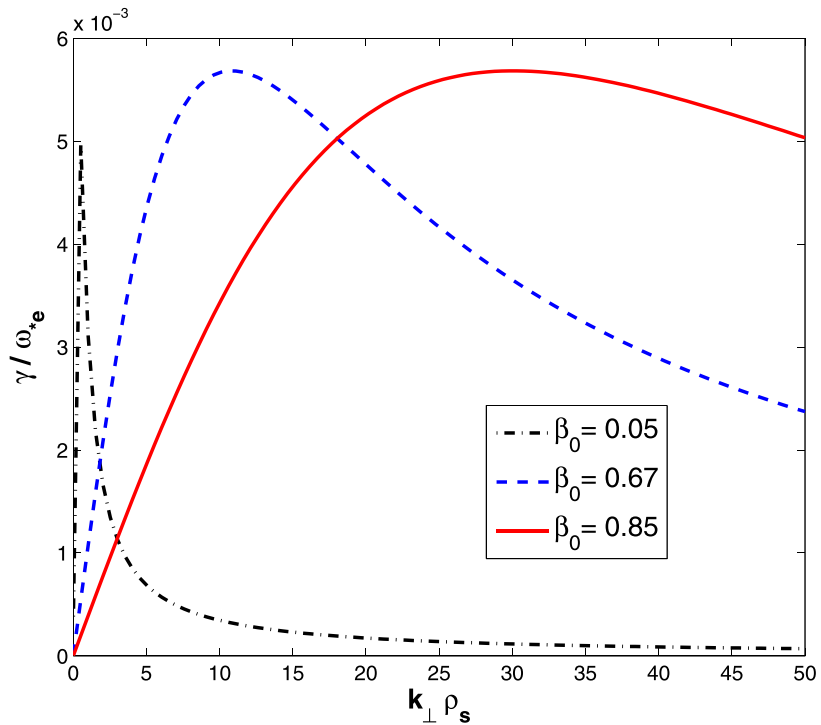

(a)

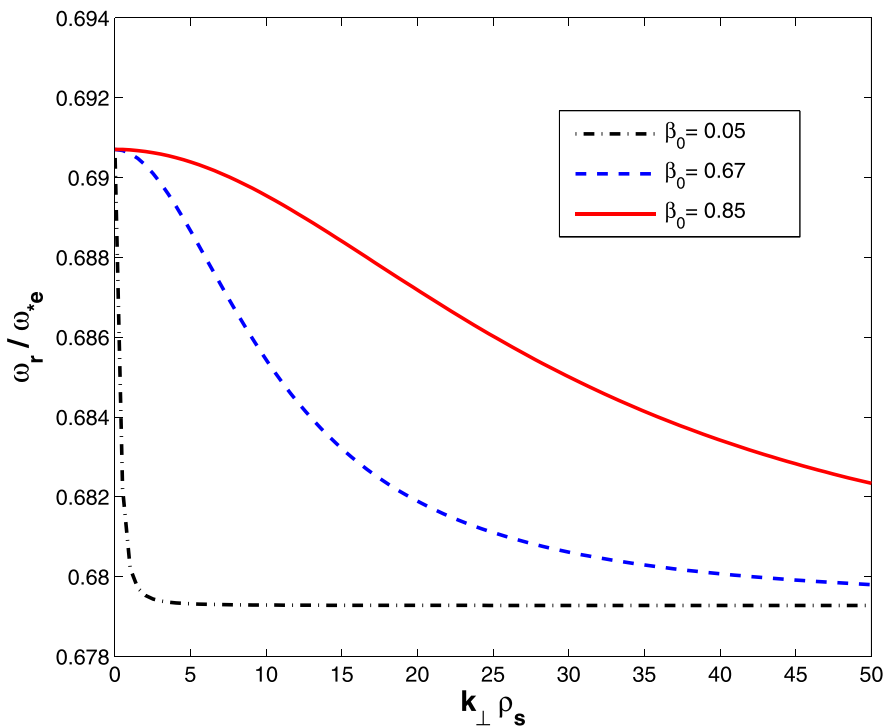

(b)

FIG. 2. Dimensionless growth rates (left) and real frequencies (right) v.s. $k_{\perp} \rho_{s}$ for different beta values.

compression. Here, all of the numerical results are obtained in the case $k_{\|} \approx 0, \eta_{\mathrm{i}}=\eta_{e}=2, \epsilon_{n}=-0.875, \tau=Z_{i}=1$, and no FLR effects. Note that the value of $\eta$ is very small in the core of FRCs because of the flat temperature profile in experiments; however, in the edge, the value of $\eta$ could be 2 or more. As mentioned in Ref. 10, the turbulent modes were not even unstable unless eta is $\sim 2$ or more, in this sense drift instabilities may grow up near the edge.

First, the influence of $\beta_{0}$ is studied and the dependencies of growth rates and real frequencies on perpendicular wave number $k_{\perp}$ are shown in Fig. 2. Here, the collisions are included. The value of $k_{\perp} \rho_{s}$ corresponding to the maximal growth rate increases with $\beta_{0}$; however, the peak growth rates rarely vary with $\beta_{0}$. In the low-beta case (red line), the maximal growth rate corresponds to $k_{\perp} \rho_{s} \sim 0.5$, which is similar to the characteristic parameter in tokamaks. When the beta is in the parameter regime of FRCs $\left(0.5<\beta_{0}<0.9\right)$, the growth rate increases quickly, reaches a maximum at $k_{\perp} \rho_{s} \sim 10-30$, and decays slowly as $k_{\perp} \rho_{s}$ increases further. It indicates that the instabilities have a broad spectrum which is close to ETG range. Usually, the maximum at $k_{\perp} \rho_{s} \sim 0.5$ is still in the regime where the expansion of the Bessel function is useful. Even in the regime of higher wave numbers $\left(k_{\perp} \rho_{s} \gg 1\right)$, our results also agree qualitatively with the results in Ref. 7 where the set of Vlasov-Maxwell equations is applied to derive the dispersion relation in FRCs

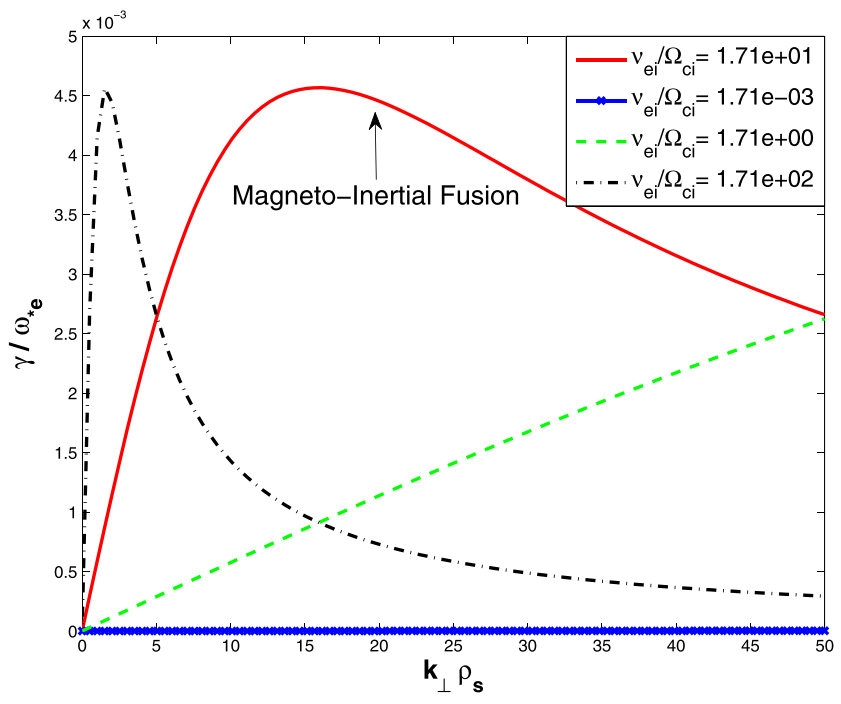

(a)

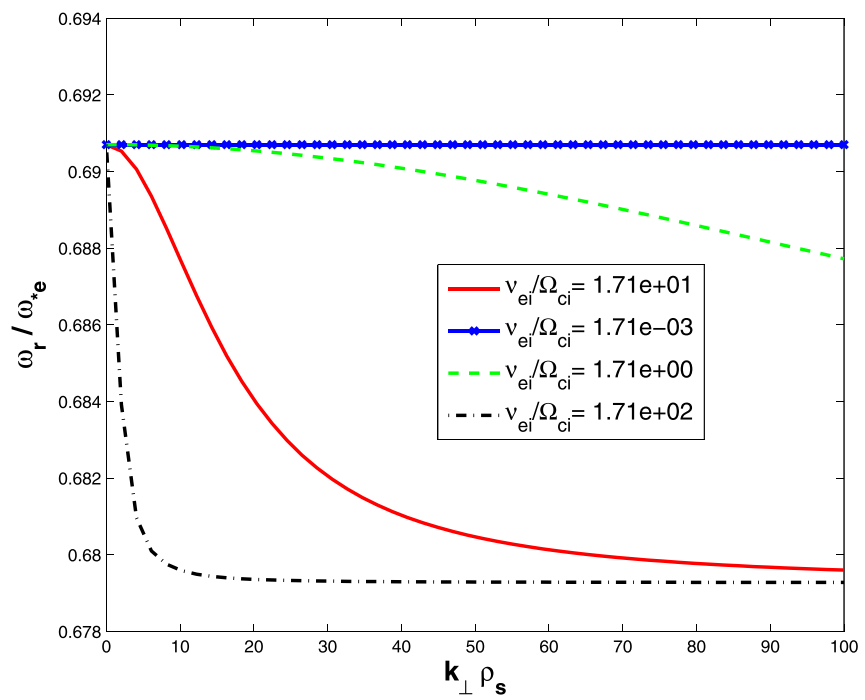

(b)

FIG. 3. Dimensionless growth rates (left) and real frequencies (right) v.s. $k_{\perp} \rho_{s}$ for different collisionalities; here, $\beta_{0}=0.76$. The red solid curve is based on the MIF parameters. 


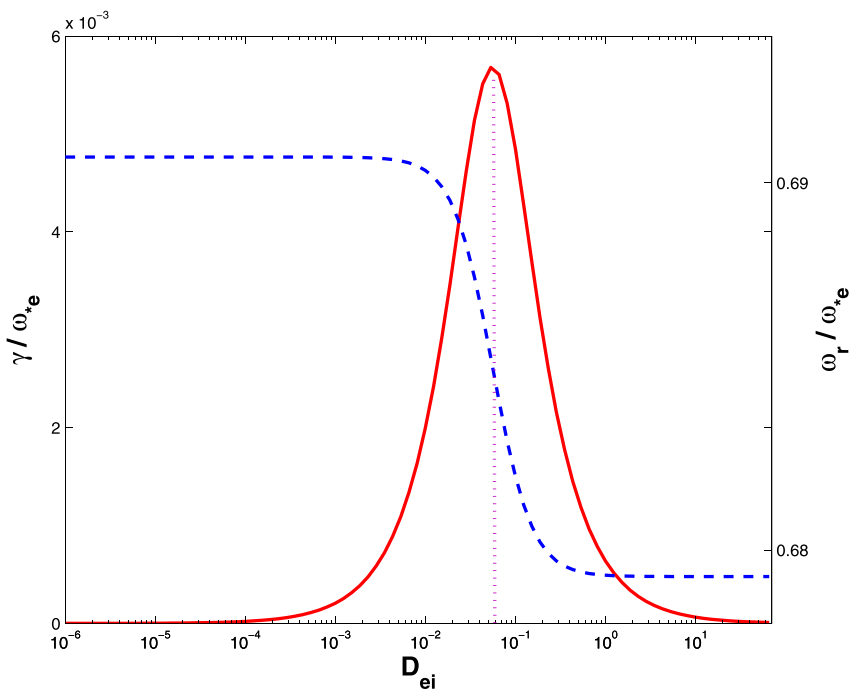

FIG. 4. Dimensionless growth rates (red solid curve) and real frequencies (blue dashed curve) as a function of normalized collisionality $\mathrm{D}_{\mathrm{ei}}$ at $k_{y} \rho_{s}=20$. In the MIF case, Dei is of the order of $10^{-1}$ (pink dotted line).

and the maximal growth rate values correspond to $k_{\perp} \rho_{s} \sim 20$.

For different collisionalities, the dispersion relations $\omega\left(k_{\perp}\right)$ are shown in Fig. 3. It is seen that the wave number corresponding to the peak growth rate becomes smaller as the collisionality increases, and the real frequency is always positive, which means that the modes propagate in the direction of electron diamagnetic drift. The results indicate that the collisions may destabilize the instability. The drift modes are almost stable at low collisionality but unstable under MIF conditions.

In order to show the effect of collisions on drift instabilities intuitively, we make a scan in collisionality (as shown in Fig. 4), and other parameters are similar to Phase I in Table I. It is shown that the growth rate almost reaches the peak when the collisionality $\mathrm{D}_{\mathrm{ei}}$ lies in the regime of MIF parameters where $\mathrm{D}_{\mathrm{ei}}$ is of the order of $10^{-1}$, and the real frequency hardly varies with the collisionality. It indicates that collisions have a destabilizing influence on drift waves under conditions of MIF. The instability mechanism may be explained in the way that the particle drifts of electrons and ions in the plane perpendicular to magnetic field become different because an ion is much heavier than an electron. This leads to charge separation effects; if a density perturbation exists, the electrons are not able to instantly neutralize the charge separation by moving along the magnetic field because of electron-ion collisions. We also could see that the growth rates tend to zero as the collisionality continues to increase, and the electrons would tend to Boltzmann distributed in the limit of infinite collisionality.

\section{CONCLUSION}

We have made the drift wave study under conditions of MIF. At the same time, we have made the study of the effect of collisions on drift instabilities both analytically and numerically. Our model is based on the two fluid equations including the collision terms, and the linear dispersion relation has been derived. By calculating the dispersion relations for different values of beta numerically, it is found that the model includes also the low-beta regime where the fastest growth rate corresponds to $k_{\perp} \rho_{s} \sim 0.5$ like the typical case of tokamak geometry. In the parameter regime of MIF, the instabilities have a broad spectrum which is close to ETG range at $k_{\perp} \rho_{s} \sim 10-30$. By making a scan in collisionality, we demonstrate the effect of collisions which could drive the instabilities in the MIF case, because the electrons cannot move along the magnetic field freely and the charge separation would not be neutralized instantly. In addition, since the collision term is introduced in the electron parallel motion equation, a finite (but small) $k_{\|}$is necessary for investigating the collisional effect; otherwise, the set of Eqs. (13) and (15) would become indeterminate. Important issues including nonlocal effects, such as magnetic shear and flow shear, need to be addressed further.

\section{ACKNOWLEDGMENTS}

This work was supported by the National Natural Science Foundation of China under Grant Nos. 11575029 and 11605013.

${ }^{1}$ M. Shimada, D. J. Campbell, V. Mukhovatov, M. Fujiwara, N. Kirneva, K. Lackner, M. Nagami, V. D. Pustovitov, N. Uckan, J. Wesley, N. Asakura, A. E. Costley, A. J. H. Donné, E. J. Doyle, A. Fasoli, C. Gormezano, Y. Gribov, O. Gruber, T. C. Hender, W. Houlberg, S. Ide, Y. Kamada, A. Leonard, B. Lipschultz, A. Loarte, K. Miyamoto, V. Mukhovatov, T. H. Osborne, A. Polevoi, and A. C. C. Sips, Nucl. Fusion 47(6), S1 (2007).

${ }^{2}$ M. K. Matzen, M. A. Sweeney, R. G. Adams, J. R. Asay, J. E. Bailey, G. R. Bennett, D. E. Bliss, D. D. Bloomquist, T. A. Brunner, R. B. Campbell, G. A. Chandler, C. A. Coverdale, M. E. Cuneo, J.-P. Davis, C. Deeney, M. P. Desjarlais, G. L. Donovan, C. J. Garasi, T. A. Haill, C. A. Hall, D. L. Hanson, M. J. Hurst, B. Jones, M. D. Knudson, R. J. Leeper, R. W. Lemke, M. G. Mazarakis, D. H. McDaniel, T. A. Mehlhorn, T. J. Nash, C. L. Olson, J. L. Porter, P. K. Rambo, S. E. Rosenthal, G. A. Rochau, L. E. Ruggles, C. L. Ruiz, T. W. L. Sanford, J. F. Seamen, D. B. Sinars, S. A. Slutz, I. C. Smith, K. W. Struve, W. A. Stygar, R. A. Vesey, E. A. Weinbrecht, D. F. Wenger, and E. P. Yu, Phys. Plasmas 12(5), 055503 (2005).

${ }^{3}$ S. C. Hsu, T. J. Awe, S. Brockington, A. Case, J. T. Cassibry, G. Kagan, S. J. Messer, M. Stanic, X. Tang, D. R. Welch, and F. D. Witherspoon, IEEE Trans. Plasma Sci. 40(5), 1287-1298 (2012).

${ }^{4}$ P. Y. Chang, G. Fiksel, M. Hohenberger, J. P. Knauer, R. Betti, F. J. Marshall, D. D. Meyerhofer, F. H. Séguin, and R. D. Petrasso, Phys. Rev. Lett. 107(3), 035006 (2011).

${ }^{5}$ M. Tuszewski, Phys. Fluids 31, 3754 (1988).

${ }^{6}$ K. F. Schoenberg, R. E. Siemon, and PoP Participants and Contributors, Los Alamos National Laboratory Technical Report No. LA-UR-98-2413, Los Alamos, NM, USA (1998).

${ }^{7}$ A. Y. Chirkov and V. I. Khvesyuk, Phys. Plasmas 17(1), 012105 (2010).

${ }^{8}$ D. P. Fulton, C. K. Lau, I. Holod, Z. Lin, and S. Dettrick, Phys. Plasmas 23(1), 012509 (2016).

${ }^{9}$ M. Tuszewski and R. K. Linford, Phys. Fluids 25(5), 765-774 (1982).

${ }^{10}$ L. C. Steinhauer, Phys. Plasmas 18(7), 070501 (2011).

${ }^{11}$ N. A. Krall and P. C. Liewer, Phys. Rev. A 4(5), 2094-2103 (1971).

${ }^{12}$ J. D. Huba, J. F. Drake, and N. T. Gladd, Phys. Fluids 23(3), 552-561 (1980).

${ }^{13}$ R. C. Davidson and N. A. Krall, Nucl. Fusion 17(6), 1313-1372 (1977).

${ }^{14}$ J. R. Soběhart and R. Farengo, Phys. Fluids B 2(12), 3206-3208 (1990).

${ }^{15}$ A. L. Hoffman and R. D. Milroy, Phys. Fluids 26(11), 3170-3172 (1983).

${ }^{16}$ H. Ji, R. Kulsrud, W. Fox, and M. Yamada, J. Geophys. Res.: Space Phys. 110, A08212, doi:10.1029/2005JA011188 (2005).

${ }^{17}$ R. Kulsrud, H. Ji, W. Fox, and M. Yamada, Phys. Plasmas 12(8), 082301 (2005). 
${ }^{18}$ Y. Wang, R. Kulsrud, and H. Ji, Phys. Plasmas 15(12), 122105 (2008).

${ }^{19}$ M. Yamada, H. Ji, S. Hsu, T. Carter, R. Kulsrud, N. Bretz, F. Jobes, Y. Ono, and F. Perkins, Phys. Plasmas 4(5), 1936-1944 (1997).

${ }^{20}$ A. W. Carlson, Phys. Fluids 30(5), 1497-1509 (1987).

${ }^{21}$ S. Okada, Y. Kiso, S. Goto, and T. Ishimura, Phys. Fluids B 1(12), 2422-2429 (1989).
${ }^{22}$ N. A. Krall, Phys. Fluids B-Plasma Phys. 1(11), 2213-2216 (1989).

${ }^{23}$ R. Farengo, P. N. Guzdar, and Y. C. Lee, Phys. Fluids B 1(11), 2181-2185 (1989).

${ }^{24}$ A. Hakim and U. Shumlak, Phys. Plasmas 14(5), 055911 (2007).

${ }^{25}$ D. D. Ryutov, Phys. Plasmas 9(9), 4085-4088 (2002).

${ }^{26}$ S. C. Guo and J. Weiland, Nucl. Fusion 37(8), 1095 (1997). 